\title{
A influência da espiritualidade e religiosidade no cuidado em saúde na percepção de estudantes da área da saúde
}

The influence of spirituality and religiosity in health care on the perception of health care students La influencia de la espiritualidad y la religiosidad en el cuidado de la salud en la percepción de los estudiantes de salud

Aparecida Samanta Lima Gonçalves ORCID: https://orcid.org/0000-0001-8228-8930 Faculdade Santo Agostinho, Brasil

E-mail: aparecidasamantalg20@gmail.com Juliana Alves Almeida ORCID: https://orcid.org/0000-0001-7568-4804 Faculdade Santo Agostinho, Brasil E-mail: juniappenf@gmail.com

Maria Aparecida Oliveira Santos ORCID: https://orcid.org/0000-0003-4815-7659 Faculdade Santo Agostinho, Brasil

E-mail: maria.aparecidaos@gmail.com

Fernando Henrique Álvares Oliveira ORCID: https://orcid.org/0000-0001-7590-1944 Faculdade Santo Agostinho, Brasil

E-mail: fernandohaoliveira@gmail.com Alanna Fernandes Paraíso

ORCID: https://orcid.org/0000-0001-7400-140X Faculdade Santo Agostinho, Brasil

E-mail: alanna.paraisoenf@gmail.com

Valdenice Ferreira dos Reis ORCID: https://orcid.org/0000-0001-5535-0401 Faculdades Unidas do Norte de Minas Gerais, Brasil

E-mail: ferreira.valdenice@yahoo.com.br Laudileyde Rocha Mota

ORCID: https://orcid.org/0000-0003-3055-1746 Faculdades Santo Agostinho, Brasil E-mail: laudileyderme@ hotmail.com

Sabrina Gonçalves Silva Pereira ORCID: https://orcid.org/0000-0001-8737-6569 Faculdade de Saúde e Humanidades Ibituruna, Brasil

E-mail: sabrinagoncalves@ funorte.edu.br

Karita Santos da Mota

ORCID: https://orcid.org/0000-0002-8862-4891 Universidade Federal de Alfenas, Brasil E-mail:karitamota@outlook.com

Nadine Antunes Teixeira

ORCID: https://orcid.org/0000-0001-7875-2921 Universidade Estadual de Montes Claros, Brasil E-mail: nadine.ateixeiraenf@gmail.com

Giuliana de Fátima Gonçalves Braga Ribeiro Escolástico ORCID: https://orcid.org/0000-0002-9072-1711 Faculdades Unidas do Norte de Minas Gerais, Brasil

E-mail: giulianabraga@hotmail.com

Mariana Stefany Cardoso Nascimento ORCID: https://orcid.org/0000-0003-2706-3653 Faculdades Unidas do Norte de Minas Gerais, Brasil

E-mail: marianastecn@outlook.com

Marcell Gonçalves Grillo

ORCID: https://orcid.org/0000-0003-4179-3142 Faculdade de Saúde e Humanidades Ibituruna, Brasil E-mail: marcellgrillogt@ hotmail.com 


\author{
Anáira Gisser de Sousa Ribeiro \\ ORCID: https://orcid.org/0000-0002-0283-4135 \\ Faculdade de Saúde e Humanidades Ibituruna, Brasil \\ E-mail: anairagiser@hotmail.com \\ Lucinei Santos Alves \\ ORCID: https://orcid.org/0000-0002-5419-546X \\ Faculdades Unidas do Norte de Minas Gerais, Brasil \\ E-mail: lucineisantos201@hotmail.com
}

\begin{abstract}
Resumo
Objetivo: Conhecer a compreensão dos acadêmicos ingressantes nos cursos das áreas da saúde em relação à espiritualidade e à religiosidade no cuidado em saúde. Metodologia: estudo descritivo de abordagem qualitativa, com amostragem intencional por conveniência. Para a concepção da pesquisa, foram coletados dados relacionados ao perfil dos entrevistados, com aplicação do instrumento FICA contendo 04 domínios de avaliação: fé/crença, importância/influência, comunidade e ação no tratamento. Em seguida, os dados foram analisados pela técnica de análise de conteúdo. Resultados: A análise dos resultados evidenciou quatro categorias: "Concepção acerca da fé/crença na vivência pessoal do indivíduo"; "A importância da religiosidade e da espiritualidade e a influência que desempenha no cotidiano das pessoas e dos profissionais da saúde"; "De que modo a comunidade religiosa pode contribuir e prestar auxílio na vida da sociedade?" Como a assistência prestada no tratamento deve ser encarada pelos profissionais da saúde e como pode afetar e/ou contribuir com a terapêutica". Conclusão: Constatou-se que grande parte dos participantes compreende a religiosidade e a espiritualidade como eficazes recursos psicológicos para o enfrentamento de situações desfavoráveis em saúde.
\end{abstract}

Palavras-chave: Assistência integral à saúde; Ciências da saúde; Espiritualidade; Religião.

\begin{abstract}
Objective: To know the understanding of academics entering health courses in relation to spirituality and religiosity in health care. Methodology: descriptive study of qualitative approach, with intentional convenience sampling. For the conception of the research, data related to the profile of the interviewees were collected, with application of the FICA instrument containing 04 evaluation domains: faith/belief, importance/influence, community and action in the treatment. Then, the data were analyzed by the content analysis technique. Results: The analysis of the results showed four categories: "Conception about faith/belief in the personal experience of the individual"; "The importance of religiosity and spirituality and the influence it plays on the daily lives of people and health professionals"; "How can the religious community contribute and assist in the life of society?" "How care provided in treatment should be seen by health professionals and how it may affect and/or contribute to therapy." Conclusion: It was found that most participants understand religiosity and spirituality as effective psychological resources for coping with unfavorable health situations.
\end{abstract} Keywords: Comprehensive health care; Health sciences; Spirituality; Religion.

\title{
Resumen
}

Objetivo: Conocer la comprensión de los académicos que ingresan a cursos de salud en relación con la espiritualidad y la religiosidad en el cuidado de la salud. Metodología: estudio descriptivo de abordaje cualitativo, con muestreo intencional de conveniencia. Para la concepción de la investigación se recogieron datos relacionados con el perfil de los entrevistados, con aplicación del instrumento FICA que contiene 04 dominios de evaluación: fe/creencia, importancia/influencia, comunidad y acción en el tratamiento. Luego, los datos fueron analizados por la técnica de análisis de contenido. Resultados: El análisis de los resultados mostró cuatro categorías: "Concepción sobre la fe/creencia en la experiencia personal del individuo"; "La importancia de la religiosidad y la espiritualidad y la influencia que tiene en la vida cotidiana de las personas y los profesionales de la salud"; "¿Cómo puede la comunidad religiosa contribuir y ayudar en la vida de la sociedad?" "Cómo la atención proporcionada en el tratamiento debe ser vista por los profesionales de la salud y cómo puede afectar y / o contribuir a la terapia". Conclusión: Se encontró que la mayoría de los participantes entienden la religiosidad y la espiritualidad como recursos psicológicos efectivos para hacer frente a situaciones de salud desfavorables.

Palabras clave: Atención integral de salud; Ciencias de la salud; Espiritualidad; Religión.

\section{Introdução}

A ligação entre religiosidade e a espiritualidade e correlação com a vida social foram, durante anos, tema de estudos por pioneiros da sociologia (Carneiro et al., 2018). Desde os primórdios, quando o homem começa a dar significado às coisas ao seu redor, buscando compreender e decifrar os acontecimentos sobrenaturais e inexplicáveis, surge a religião, como forma de evasão de o sujeito ter em que ou quem depositar a sua fé. A religiosidade é vista por seguir os preceitos de uma determinada doutrina, um conjunto de práticas, credos, hábitos e ritos os quais encerram um apelo humano pelo mistério do mundo. Cria, 
assim, uma visão da realidade que é sagrada, abrangente e sobrenatural (Koenig, 2012; Jardim, 2015; Dias, et al., 2020).

A espiritualidade pode ser entendida como o aspecto humano que diz respeito ao modo como os indivíduos buscam e expressam sentido e propósito e o modo como experienciam conexão com o momento, consigo mesmo, com os outros, com a natureza e com o transcendente ou sagrado" (Puchalski, et al., 2009).

O gradativo número de pesquisas associadas à religiosidade e à espiritualidade aplicadas nos diversos setores nas áreas das ciências da saúde, vem sendo cada vez mais discutido no meio científico (Panitizet, et al., 2018). A busca por novas pesquisas acerca do tema evidencia o distinto querer por estratégias e soluções que minimizem o sofrimento humano, propondo métodos e formas que visam facilitar a sua abordagem à comunidade. O estudo acerca da percepção no âmbito acadêmico, profissional e aos pacientes reflete a importância da fé e da espiritualidade na vida pessoal coletiva e no tratamento (Dezorzi, \& Crosseti, 2008; Lucchetti, et al., 2010; Tavares, et al., 2018).

Estudos retratam que a espiritualidade e a religiosidade são fatores que afetam diretamente desde o processo de diagnóstico até a convivência diária, adesão ao tratamento e a terapêutica (Gomes, \& Santos, 2013). Nesse sentido, o estudo propôs como objetivo conhecer a percepção de acadêmicos do curso de graduação em enfermagem, fisioterapia e farmácia sobre a religiosidade, a espiritualidade e a saúde.

\section{Metodologia}

Trata-se de um estudo descritivo de abordagem qualitativa, segundo os preceitos de Minayo (2013). A coleta de dados foi realizada em novembro de 2018 com 40 (quarenta) acadêmicos do $3^{\circ}$ período do curso de enfermagem, fisioterapia e farmácia, sendo 12 (doze) acadêmicos de enfermagem, 10 (dez) de fisioterapia e 18 (dezoito) de farmácia, selecionados por meio de amostragem por conveniência. A coleta procedeu-se em uma instituição privada de ensino superior localizada no Norte de Minas Gerais, Brasil. Compuseram o estudo acadêmicos que aceitaram voluntariamente participar da pesquisa após leitura e assinatura do Termo de Consentimento Livre e Esclarecido (TCLE).

Coletaram-se variáveis relacionadas ao curso, procedência, gênero, idade, estado civil, cor/raça e afiliação religiosa. No segundo momento, foi aplicado o questionário FICA validado por Puchalski \& Romer (2000), contendo 12 questões norteadoras em que cada sigla corresponde a um conceito descrito a seguir:

\section{FÉ/CRENÇA:}

Você se considera religioso ou espiritualizado?

Você tem crenças espirituais ou religiosas que te ajudam a lidar com problemas?

Se não: o que te dá significado na vida?

\section{IMPORT NCIA/INFLUÊNCIA:}

Que importância você dá para a fé ou crenças religiosas em sua vida?

A fé ou crenças já influenciaram você a lidar com estresse ou problemas de saúde?

Você tem alguma crença específica que pode afetar decisões médicas ou o seu tratamento?

\section{COMUNIDADE:}

Você faz parte de alguma comunidade religiosa ou espiritual?

Ela te dá suporte, como?

Existe algum grupo de pessoas que você "realmente" ama ou que seja importante para você?

AÇÃO NO TRATAMENTO:

Como você gostaria que o seu médico ou profissional da área da saúde considerassem a questão religiosa/ espiritualidade no seu tratamento? 
Indique, remeta a algum líder espiritual/religioso.

As perguntas eram abertas de maneira a permitir aos universitários expressarem suas opiniões e percepções referentes ao tema. Com a finalidade de garantir o sigilo e o anonimato, os acadêmicos foram identificados pela letra P (participante), seguida de um número que representava a ordem de ingresso no estudo. As transcrições das entrevistas foram realizadas na íntegra. A análise dos dados ocorreu em etapas fundamentais, conforme instruções para análise de conteúdo como descrito por Bardin, (2011). Para análise dos dados sociodemográficos dos participantes, foi elaborada uma tabela descritiva. A última etapa foi a integração das categorias em temas maiores, as quais foram descritas em quatro temáticas descritas neste estudo.

Atendendo às Diretrizes e Normas Regulamentadoras do Conselho Nacional de Saúde, por meio da Resolução 466/2012, foi submetido ao Comitê de Ética em Pesquisa e autorizado por meio do parecer nº 2.525.936.

\section{Resultados e Discussão}

Após a delimitação da amostra e aplicação de critérios de inclusão, o presente estudo teve como participantes da pesquisa 40 acadêmicos dos cursos de enfermagem, fisioterapia e farmácia. A partir da análise dos dados sociodemográficos fornecidos, foi possível traçar o perfil da amostra, como mostra a Tabela 1. Constata-se um predomínio de participantes do sexo feminino, com idades variantes entre 19 a 29 anos, e solteiros (as). Na variável cor/raça, observou-se que a maioria se autodeclarou da raça parda. Quanto à afiliação religiosa, há um predomínio de católicos.

Tabela 1 - Perfil sociodemográfico dos acadêmicos, 2018.

\begin{tabular}{lll}
\hline Variável & $\mathbf{n}$ & $\mathbf{\%}$ \\
\hline Idade & 34 & $85 \%$ \\
19 a 29 anos & 6 & $15 \%$ \\
30 a 49 anos & & $70 \%$ \\
Sexo & 28 & $30 \%$ \\
Feminino & 12 & $70 \%$ \\
Masculino & & $30 \%$ \\
Estado Conjugal & 28 & \\
Solteiro (a) & 12 & \\
Casado (a) & & $22,5 \%$ \\
Cor/Raça & & $67,5 \%$ \\
Branco & 9 & $10 \%$ \\
Pardo & 97 & \\
Preto & 27 & $57,5 \%$ \\
Afiliação Religiosa & 4 & $2,5 \%$ \\
Católica & & $2,5 \%$ \\
Espírita & $27,5 \%$ \\
Evangélica Pentecostal & 23 & $10 \%$ \\
Evangélica de Missão & 1 & $\mathbf{1 0 0 \%}$ \\
\hline Tonhum & 1 & \\
\hline
\end{tabular}

Fonte: Autores.

De acordo com o último censo demográfico, realizado pelo Instituto Brasileiro de Geografia e Estatística (2010), a maior parte da população brasileira é considerada cristã, de afiliação católica, contudo, em comparação aos resultados apresentados no ano 2000, há a diminuição do número de católicos e o crescente aumento de evangélicos em diversas áreas do Brasil. A pesquisa ainda indica o aumento do total de espíritas, dos que se declararam sem religião, e do conjunto pertencente às outras religiões. Tais dados revelam o crescimento da diversidade dos grupos religiosos (IBGE, 2012).

Com base na análise dos questionários e na verificação da percepção dos estudantes a respeito do tema, o estudo foi dividido nas seguintes categorias de acordo com o questionário FICA, sendo elas: "Concepção acerca da Fé/Crença na vivência 
pessoal do indivíduo"; "A importância da religiosidade e espiritualidade e a influência que desempenham no cotidiano das pessoas e dos profissionais da saúde"; "De que modo a comunidade religiosa pode contribuir e prestar auxílio na vida da sociedade?"; "Como a assistência prestada no tratamento deve ser encarada pelos profissionais da saúde e como isso pode afetar e/ou contribuir para a terapêutica".

Concepção acerca da Fé/Crença na vivência pessoal do indivíduo

Embora as ideologias sobre religiosidade e espiritualidade sejam utilizadas como sinônimos, possuem conceitos distintos. Desse modo, tais dificuldades surgem na literatura da área, que enfatiza a familiaridade e a discrepância existentes entre os termos. Além disso, em geral, um serve de complemento ao outro, ainda que apresentem distinção entre eles (Zanetti et al., 2018). Algumas vertentes tendem a ser exploradas no contexto da fé/crença: "Você se considera religioso ou espiritualizado?" Os depoimentos abaixo demonstram a falta de clareza e o pouco conhecimento em relação aos conceitos refletindo na tentativa dos participantes para exprimirem o seu ponto de vista e entendimento:

"Sim, me considero espiritualizado, porém não religioso "(P1).

"Sim, pois compartilho de uma fé e uma religião, acima de tudo acredito em Deus" (P2).

"Não me considero uma pessoa religiosa, pois não pratico nenhuma religião. Espiritualizado às vezes" (P5).

"Não. Acredito em Deus, mas não me considero uma pessoa religiosa" (P21).

"Sim, pois é em Deus que busco forças para continuar" (P27).

"Sim, através da minha espiritualidade me sinto fortificada a cada dia" (P40).

Vários estudos acerca do tema demonstram que pessoas que praticam ou acreditam na religiosidade e espiritualidade desenvolvem qualidade de vida e diminuem a predominância de doenças. Não há somente uma maneira de abordar a espiritualidade. Comumente, a abordagem sobre essa temática é realizada naturalmente e está associada às heranças culturais de cada profissional da saúde (Lucchetti, et al., 2010).

A religiosidade e a espiritualidade são consideradas símbolos, guias e/ou idéias presentes no dia a dia da sociedade, ou seja, fazem parte do processo da pessoa humana em toda a sua gênese. Esse vínculo de dogmas aponta que a experiência religiosa faz parte de uma vida com sentido, em que o ser humano explora a força de sua dimensão espiritual, buscando, assim, forças para lidar com acontecimentos ou fatos (Oliveira, \& Junges, 2012).

Entende-se, portanto, que quem acredita o faz porque está coerentemente seguro daquilo ou em quem crê. Ou seja, esse é um crescimento progressivo. $\mathrm{O}$ crer é proveniente de inúmeras experiências usufruídas em torno do que se vai acreditar, quer por conhecimento, convívio, leituras, estudos, preceitos e reflexões. Quando se acredita numa pessoa, em conceitos, filosofias, religiões, é porque há segurança.

Dentre as perspectivas de religiosidade abordadas, os acadêmicos utilizaram palavras, como expectativa em Deus, acreditar e ter pensamento positivo, reforçando achados da literatura, os quais são vistos como uma conexão com seu Deus, além de trazer a ideia de fé (Bousso et al., 2010).

A espiritualidade é o mecanismo da individualidade de cada ser humano, uma parte de sua identidade e da sua finalidade como ser, à luz da sua história, experiência e aspiração. Por isso, a expectativa em Deus faz com que o acreditar e o ter pensamentos positivos contribuam para as superações das falhas éticas e morais. A religião, que tem relação com o sagrado, portanto, gera alívio ao sofrimento, na medida em que propicia mudanças na visão como o paciente e a comunidade compreendem o contexto da doença. 
A importância da religiosidade e da espiritualidade e a influência que desempenha no cotidiano das pessoas e dos profissionais da saúde

A religião pode influenciar de maneira positiva na saúde e também na prevenção e na recuperação de problemas, seja no contexto pessoal ou na saúde do indivíduo, possibilitando mudanças de rotinas, hábitos e comportamentos (Zerbetto, et al., 2014).

Ao questionar "Que importância você dá à fé ou crenças religiosas em sua vida?" foi marcante por alguns estudantes que a fé ou as crenças religiosas exercem uma função essencial e fundamental para o equilíbrio e harmonia entre as dimensões do ser humano:

“Total importância nos seres humanos, precisamos de uma base para sobreviver à fé em Jesus Cristo é crucial "(P2).

"Não seríamos nada sem fé, pois uma pessoa sem fé é uma pessoa sem Deus. Afinal homem nenhum jamais viu ou verá a ele. É pela fé que o conhecemos" (P5).

"Tenho uma fé muito grande, onde com ela, busco me fortalecer em minha vida" (P11).

"Considero algo muito importante em que baseio a maior parte do que acontece na minha vida" (P12).

"A fé na minha vida é essencial, as crenças religiosas não consideram que sejam tão fundamentais assim” (P32).

A religião está vinculada à vida do homem desde a antiguidade. Conforto para os sofrimentos e confiança que a morte não seja o fim da vida são questões a que o homem procura constantemente responder. As convicções religiosas exprimem-se como mecanismo para se obterem as explicações que se esperam, constituem um meio de responder às dúvidas existentes (Peterle, 2014).

A religião, por meio da história, tem sua influência na vida das sociedades, em seu modo de pensar e de agir. Sua relação com o homem tem o sentido de condutor, contribuindo para seu crescimento e para seu desenvolvimento como ser social e humano. As religiões são, sem dúvida, um instrumento de controle e de coesão dos grupos sociais. A fé é o instrumento que motiva e dá sentido à vida do homem em todos os tempos históricos. Dessa forma é que se entende o porquê de respostas tão categóricas de alguns entrevistados, quando dizem que:

"Não seríamos nada sem fé, pois uma pessoa sem fé é uma pessoa sem Deus. Afinal homem nenhum jamais viu ou verá a ele. É pela fé que o conhecemos; Tenho uma fé muito grande, onde com ela, busco me fortalecer em minha vida" (P36).

Nota-se que há uma dimensão maior, responsável pelo controle sobre as contingências presentes na vida, capacitando o indivíduo a lidar com os acontecimentos de forma mais tranquila, confiante, de maneira a reduzir o número de transtornos, tais como depressão, estresse e ansiedade, dentre outros (Fornazari, \& Ferreira, 2010). Quanto maior a participação do indivíduo ao meio religioso, menor é a sintomatologia depressiva manifestada e que, quanto maior o hábito de orações dos indivíduos, menos ampliada são os sintomas depressivos dos indivíduos (Dias, 2011).

Em relação à influência da fé sobre os pacientes, Koenig (2005), em seus estudos, diz que a fé é uma força poderosa que ajuda o indivíduo a superar fases difíceis da doença e ainda reforça a confiança em suas próprias energias e o propósito de continuar vivendo. Além de proporcionar melhores resultados no tratamento médico e de acelerar a recuperação do paciente, a religiosidade, seja ela cristã, judaica, muçulmana entre outras, pode estar associada a uma maior longevidade. 
De que modo a comunidade religiosa pode contribuir e prestar auxílio na vida da sociedade?

A religião foi descrita como um conjunto de dogmas e normas organizacionais. A religiosidade ainda se apresenta como a expressão ou prática do crente que pode estar relacionada a uma instituição religiosa (Silva, \& Siqueira, 2009). Dezenas de estudos mostram que fiéis são mais felizes, vivem mais e são mais agradáveis. Mas também não há mais dúvidas de que é possível reproduzir esses efeitos em ateus e pessoas sem religião (Lisboa, 2014).

O estudo de Lisboa (2014) em seus resultados traz exatamente o que se questiona sobre pertencer ou não a alguma comunidade religiosa/espiritual e sobre o suporte recebido. Você faz parte de alguma comunidade religiosa ou espiritual? Percebeu-se uma variabilidade nas opiniões, em que alguns dos participantes afirmaram participar ou fazer parte de grupos ou comunidades religiosas ou espirituais, enquanto outros negaram participar ou fazer parte destes.

\author{
"Não faço parte de comunidade religiosa" (P1). \\ "Sim, frequento a igreja" (P2). \\ "Sim, comunidade católica" (P18). \\ “No momento não" (P24). \\ "Sim, mas não frequento" (P39).
}

Os dados do censo demográfico, realizado no ano de 2010, evidencia que parcela superior a 90\% da população brasileira possui alguma denominação religiosa e grande parte da população brasileira possui alguma forma de expressão espiritual ou envolvimento religioso (Braghetta, et al., 2011; IBGE, 2012)

A igreja não se refere apenas ao edifício onde se celebram os ritos religiosos. Trata-se da comunidade ou do conjunto de fiéis que professam a mesma fé. Portanto, o termo igreja não se refere apenas a uma instituição com dogmas, ritos e hierarquias definidos, mas, sim, como um grupo de indivíduos na sociedade que se inter-relacionam mediados pela mesma crença (Farinasso, \& Labate, 2012).

As diversas religiões, existentes no mundo desempenham prestígio na vida dos seres humanos, no entanto, todas tentam suprir algo superior que vem de um ser supremo. A religião, para alguns, é um mundo necessário, um estilo e sentido da vida. Para outros, um complemento nada necessário. Desde o princípio, cientistas e estudiosos objetivam compreender como surgiu o homem e o motivo pelo qual todas as coisas acontecem. É certo dizer que, desde o passado, no período pré-histórico, antes do evangelho e de Jesus Cristo, no período de formação do mundo, segundo cientistas, o homem já acreditava em um ser supremo e sempre usava da fé (Boff, 2004).

A compreensão religiosa e/ou espiritual traz conceito e desígnio para a vida das pessoas, sobretudo, quando se encontram fragilizados pela dor. Esse envolvimento pode intervir em decisões médicas, tendo que ser identificado na anamnese. Encontram-se relatos de dificuldades na tomada de história espiritual por parte dos médicos, embora, haja predisposição dos pacientes em compartilhar suas crenças e comprovação científica da eficácia terapêutica da religiosidade/espiritualidade na saúde física e mental (Abuchaim, 2018).

Nesse sentido, a religiosidade e a espiritualidade podem ter êxito protetor sobre a saúde e influenciar a saúde física e mental de diferentes formas, por exemplo, por meio de princípios e do desestímulo a atitudes prejudiciais à saúde, como abuso de álcool e drogas, comportamento violento ou sexual de risco; por meio do uso de crenças religiosas como forma de lidar com situações adversas, como uma doença, e por meio da criação de uma rede de suporte social (Braghetta, et al., 2011; Brandão, et al., 2020).

Questionados em como a religiosidade e a espiritualidade oferecem suporte, surgiu a seguinte indagação: Comunidade como igrejas, templos, centros, grupos de apoio são fontes de suporte importantes? Mesmo os estudantes que não possuem 
nenhuma religião ou não estão inseridos em algum meio, consideram de fundamental importância a participação de comunidades, grupos e principalmente igrejas.

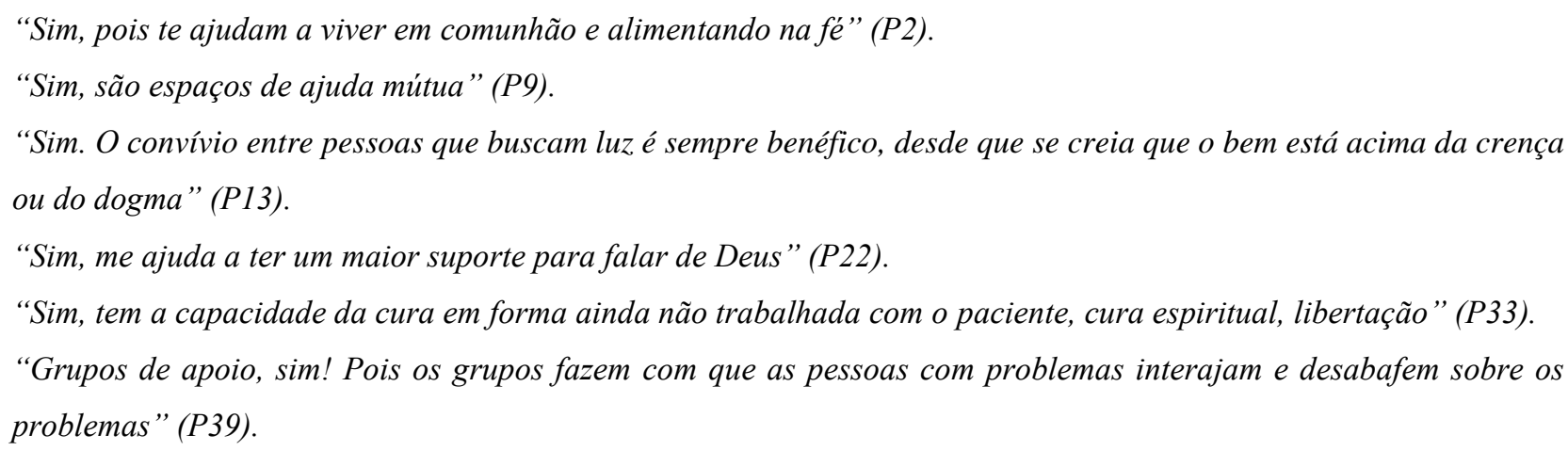

Nesse contexto, é importante discutir que a compreensão religiosa ou religiosidade compreende os procedimentos, ações, princípios, crenças, sentimentos e experiências instigados pelo contexto religioso, desdobrando-se em três dimensões: religiosidade organizacional (RO), religiosidade não organizacional (RNO) e religiosidade intrínseca (RI) (Santos, \& Abdala, 2014).

A religiosidade organizacional compreende os comportamentos religiosos que decorrem no âmbito da instituição religiosa (como a frequência às atividades religiosas formais) e a atuação de cargos ou funções religiosas. Já a religiosidade não organizacional compreende os comportamentos religiosos privados ou informais, ou seja, que se dão fora do contexto da instituição religiosa, sem local e tempo fixos e sem seguirem formas litúrgicas predispostas, podendo se manifestar individualmente ou em pequenos grupos familiares e informais. A religiosidade intrínseca analisa de que forma a religião pode provocar ou incitar comportamentos, decisões e, de forma geral, a vida do sujeito. Refere-se a uma dimensão subjetiva, de quanto ou como o indivíduo percebe a importância da religião em sua vida (Santos, \& Abdala, 2014).

Como a assistência prestada no tratamento deve ser encarada pelos profissionais da saúde e como isto pode afetar e/ou contribuir a terapêutica

Outro aspecto que emergiu das falas dos estudantes foi à visão religiosa e espiritual, quanto à ação no tratamento: Como você gostaria que o seu médico ou profissional da área da saúde considerasse a questão religiosa/espiritualidade no seu tratamento? A maior parte dos participantes possui opiniões em comum de que gostariam que o tratamento fosse baseado no respeito, independentemente da religião ou crença, e que os profissionais engajados no processo de diagnóstico e terapêutica buscassem medidas e até mesmo consentimento para que se realize o tratamento de modo a salvar vidas ou evitar possíveis danos com a não adesão. Outra questão apresentada foi a citação de que fé e Jesus são a base da cura e de que isso pode interferir positivamente na recuperação.

"Gostaria que tivesse mais importância, pois a crença em Jesus é a base da cura "(P2).

"A medicina já tem sofrido modificações a respeito desse assunto, profissionais têm se aprofundado, quando se deparam com curas misteriosas ou questões religiosas, como as transfusões sanguíneas na religião (testemunhas de Jeová), primeiro respeitar, depois "acreditar" que a fé pode realizar curas" (P9).

"De forma normal, independente da minha religião" (P13).

"Com respeito. Se a fé do paciente pode produzir um campo energético positivo, sempre vai interferir positivamente também em sua recuperação" (P15). 
"Respeitasse a minha crença e aplicasse para auxiliar no processo "(P20).

"Apenas que ele respeitasse, mesmo que não tenha a mesma crença" (P21).

“Com respeito, pois se a pessoa não quer fazer o tratamento por algum motivo ou religião deve-se respeitar, mas se o caso é grave buscar uma maneira de fazer o tratamento com o consentimento da família" (P37).

A agregação no âmbito de religiosidade e saúde possui vínculos históricos: durante a maior parte da história da humanidade, o fascínio, a religião e a cura quase sempre andaram juntos. No entanto, a linguagem médico-científica tem se determinado pela busca da neutralidade e da objetividade, bem como pela indiferença às particularidades culturais presentes no relacionamento com os pacientes, aí incluída a religiosidade (Pinto, \& Falcão, 2013).

\section{Conclusão}

O estudo procurou conhecer sobre a influência da espiritualidade e da religiosidade no cuidado em saúde na percepção dos acadêmicos da área da saúde. Os resultados corroboram para a disposição próspera de reconhecer fatores socioculturais no processo da assistência em saúde inclusa à religiosidade/espiritualidade. Constatou-se que grande parte do grupo de acadêmicos investigados compreende a crença religiosa e a espiritualidade como eficazes recursos para o aprendizado de situações na área da saúde. Percebe-se que a temática com relação à espiritualidade e à religiosidade sempre foi e continua fazendo parte de um assunto abrangente e complexo.

No presente, vive-se em um mundo em que o ser humano obtém motivações de vários ângulos e que influenciam completamente com a sua forma de portar-se e de pensar e até em seu estilo de vida. E a religião, como um destes aspectos, está impregnada no modo de ser das pessoas, na vivência familiar, colaborando para prováveis transformações comportamentais. Nota-se, por meio das respostas, que a espiritualidade e a religiosidade são muito relevantes na vida dos estudantes entrevistados, justificando a necessidade de uma maior atenção à questão espiritual e religiosa do paciente na assistência nos serviços de saúde. Sugere-se a realização de novos estudos que avaliem a inserção dessas temáticas no currículo acadêmico para aperfeiçoar as práticas em saúde.

\section{Referências}

Abuchaim, S. C. B. (2018). Espiritualidade/Religiosidade como Recurso Terapêutico na Prática Clínica: concepção dos Estudantes de Graduação em Medicina da Escola Paulista de Medicina - Universidade Federal de São Paulo. São Paulo, 2018. Dissertação (Mestrado em Ciência da Saúde CEDESS) - Universidade Federal de São Paulo.

Bardin, L. (2011). Análise de conteúdo. Edições 70, 229.

Boff, L. (2004). Novas Fronteiras da Igreja. Ed Versus, 102.

Braghetta, C. C., Lucchetti, G., Leão, F. C., Vallada, C., Vallada, H., \& et al. (2011). Aspectos éticos e legais da assistência religiosa em hospitais psiquiátricos. Revista de Psiquiatria Clínica, 38(5), 186-193.

Bousso, R. S, Misko, M. D, \& Serafim, T. (2010). The relationship between religion, illness and death in life histories of family members of children with lifethreatening diseases. Revista Latino-Americana de Enfermagem Ribeirão Preto, 18 (2), 156-162.

Brasil. (2010). Ministério do Planejamento, Orçamento e Gestão. Instituto Brasileiro de Geografia e Estatística- IBGE. Censo Demográfico 2010. Características gerais da população, religião e pessoas com deficiência. 89- 105 .

Brandão, J. L. et al. (2020). Espiritualidade e Religiosidade no contexto da integralidade da assistência: reflexões sobre o cuidado integral em saúde e enfermagem. Research, Society and Development, 9(10), 1-22.

Carneiro, L. C., Serafim, M. C., \& Tezza, R. (2018). Uma Análise Bibliométrica da Relação entre Ética e Espiritualidade/Religiosidade nas Organizações. Revista Interdisciplinar de Gestão Social -RIGS, 7(1), 43-166.

Dezorzi, L. W., \& Crossetti, M. G. O. (2008). A espiritualidade no cuidado de si para os profissionais de enfermagem em terapia intensiva. Revista LatinoAmericana de Enfermagem, 16(2), 212-217. 
Research, Society and Development, v. 11, n. 3, e46811325300, 2022

(CC BY 4.0) | ISSN 2525-3409 | DOI: http://dx.doi.org/10.33448/rsd-v11i3.25300

Dias, C. M. B. L. C. (2011). Religiosidade intrínseca e extrínseca: Implicações no bem-estar subjetivo de adultos de meia idade. 2011. 51f. Dissertação (Mestrado Integrado em Psicologia) - Universidade de Lisboa, Lisboa.

Dias, F.A. et al. (2020). Espiritualidade e saúde: uma reflexão crítica sobre a vida simbólica. Research, Society and Development, 9(5), 1-24.

Farinasso, A. L. D. C., \& Labate, R. C. (2012). Luto, religiosidade e espiritualidade: um estudo clínico-qualitativo com viúvas idosas. Revista Eletrônica de Enfermagem, 14(3), 588-595.

Fornazari, S. A., \& Ferreira, R. E. R. (2010). Religiosidade/Espiritualidade em Pacientes Oncológicos: Qualidade de Vida e Saúde. Psicologia: Teoria e Pesquisa, 26(2), 265-272.

Gomes, A. M. T. \& Santo, C. C. D. E. (2013). A espiritualidade e o cuidado de enfermagem: desafios e perspectivas no contexto do processo saúde doença. Revista Enfermagem UERJ, 21(2), 261-262.

Instituto Brasileiro de Geografia e Estatística. (2012). Censo Brasileiro de 2010. IBGE.

Jardim, G. M. (2015). Religião numa visão sociológica e científica. 2015. 15f. Trabalho de Conclusão de Curso. Universidade Federal de Santa Maria.

Koenig, H. G. (2005). Saúde e Espiritualidade. Revista Vivasaúde, 15, (82).

Koenig, H. G. (2012). Medicina, religião e saúde: o encontro da ciência e da espiritualidade. Porto Alegre (RS): $L \& P M$.

Lisboa, S. (2016). A ciência da fé. Revista Super Interessante. https://super.abril.com.br/ciencia/a-ciencia-da-fe/

Lucchetti, G., Granero, A. L., Bassi, M. R., Latorraca, R., \& Nacif, P. A. S. (2010). Espiritualidade na Prática Clínica: o que o Clínico deve Saber? Revista da Sociedade Brasileira de Clínica Médica, 2(8), 54-158.

Minayo, M.C.S. O desafio do conhecimento: Pesquisa qualitativa em saúde. Hucitec, 2013.

Oliveira, M. R. D., \& Junges, J. R. (2012) Saúde mental e espiritualidade/religiosidade: a visão de psicólogos. Estudos de Psicologia, 17(3), 469-476.

Panitiz, O. G., et al. (2018). Instrumento de abordagem da espiritualidade na prática clínica. Acta médica - ligas acadêmicas, 39 (1), $37-45$.

Peterle, E. A. (2014). Importância da Religiosidade. Universo UFES.

Pinto, A. N., \& Falcão, E. B. M. (2013). Religiosidade no contexto médico: entre a receptividade e o silêncio. Revista Brasileira de Educação Médica, 38(1), $38-46$.

Pinto, E. B. (2009). Espiritualidade e Religiosidade: Articulações. Revista de Estudos da Religião - REVER, 1(1),68-83.

Puchalski, C., \& Romer A. L. (2000). Taking a spiritual history allows clinicians to understand patients more fully. Journal of Palliative Medicine, 3(1), 129137.

Puchalsky, C., et al. (2009). Improving the Quality of Spiritual Care as a Dimension of Palliative Care: The Report of the Consensus Conference. Journal of Palliative Medicine, 12(10), 885-904.

Santos, N. C. D. S., \& Abdala, G. A. (2014). Religiosidade e qualidade de vida relacionada à saúde dos idosos em um município na Bahia, Brasil. Revista Brasileira de Geriatria e Gerontologia, 17(4), 795-805.

Silva, R. R. D., \& Siqueira, D. (2009). Espiritualidade, religião e trabalho no contexto organizacional. Psicologia em Estudo, 14(3), 557-564.

Tavares, M. D. M. (2018). Espiritualidade e religiosidade no cotidiano da enfermagem hospitalar. Revista de Enfermagem UFPE on line, 12(4), $1097-1102$.

Zanetti, G. C. et al. (2018). Percepção de acadêmicos de medicina e de outras áreas da saúde e humanas (ligadas à saúde) sobre as relações entre espiritualidade, religiosidade e saúde. Revista Brasileira de Educação Médica, 1(42), 65-72.

Zerbetto, S. R. et al. (2017). Religiosidade e espiritualidade: mecanismos de influência positiva sobre a vida e tratamento do alcoolista. Escola Anna Nery, 21(1), $1-8$. 